\author{
О.Г. Руденко, О.О. Безсонов, Н.М. Сердюк, К.О. Олійник, О.С. Романюк
}

Харківський національний університет радіоелектроніки, Харків

\title{
РОБАСТНА ІДЕНТИФІКАЦІЯ ОБ'ЄКТІВ НА ОСНОВІ МІНІМІЗАЦІЇ КОМБІНОВАНОГО ФУНКЦІОНАЛУ
}

Розглянуто задачу ідентифікачії параметрів лінійного об'єкта за наявністю негаусівських завад на основі мінімізації комбінованого функціоналу, який поєднує властивості МНК та МНМ та забезпечує робастність одержуваних оцінок. Досліджено статистичні властивості градієнтного алгоритму ідентифікаиіï, в результаті чого визначено умови його збіжності в середньому і середньоквадратичному. Для дослідження властивостей в алгоритму використано функиії Ляпунова. Отримано аналітичні оцінки неасимпотичних та асимптотичних значень помилки оцінювання параметрів і точності ідентифікації. Показано, щзо ці значення помилки очінювання і точності ідентифікації залежать від вибору параметра змімування. Отримані оцінки є досить загальними і залежать від статистичних характеристик корисних сигналів $i$ завад. Тому для їх практичного застосування слід скористатися оцінками цих параметрів. Результати експериментального дослідження властивостей алгоритму при ідентифікації об'єкту, вихід якого вимірюється з незалежним шумом з розподілом Релея, некорельованим з сигналами, свідчать про ефективність підходу, щзо розвивається. Отримані в роботі оцінки дозволяють досліднику при вирішенні практичних завдань попередньо оцінити можливості досліджуваного алгоритму і ефективність його застосування.

Ключові слова: комбінований критерій, градієнтний алгоритм, параметр зважування, рекурентна процедура, асимптотична оцінка, точність ідентифікації.

\section{Вступ}

Постановка проблеми. В основі багатьох задач обробки інформації (обробка та фільтрація складних сигналів, ідентифікація об'єктів та керування ними, прогнозування часових послідовностей тощо) лежить задача побудови моделі виду:

$$
y(k)=\theta^{* T} x(k)+\xi(k),
$$

де $y(k)$ - спостережуваний вихідний сигнал; $x(k)=\left(x_{1}(k), x_{2}(k), . . x_{N}(k)\right)^{T}$ - вектор вхідних сигналів $N \times 1 ; \quad \theta^{*}=\left(\theta_{1}^{*}, \theta_{2}^{*}, . . \theta_{N}^{*}\right)^{T}$ - вектор шуканих параметрів $N \times 1$; $\xi(k)$ - завада, і зводяться до мінімізації деякого наперед обраного функціоналу якості (критерію ідентифікаціі).

Найбільш широко використовуваний на практиці квадратичний функціонал призводить до різних алгоритмів ідентифікації, що дозволяє отримати оцінки шуканого вектора $\theta^{*}$ при нормальних розподілах завади, тобто $\xi(k) \sim N\left(0, \sigma_{\xi}^{2}\right)$.

Засноване на цьому припущенні МНК-рішення $\epsilon$ асимптотично оптимальним з мінімальною дисперсією в класі незміщених оцінок. Однак це припущення, як правило, не $\epsilon$ вірним в реальних умовах, тому що майже завжди апріорна інформація про розподіл зазвичай недоступна або завада є засміченою негаусівським шумом, через що деякі вимірювання видалені на відносно велику відстань від основного обсягу даних і утворюють так звані “хвости”. Нестійкість оцінки МНК при наявності таких завад послужила підставою для розвитку альтернативного, робастного оцінювання в статистиці, метою якого і стало виключення впливу помилок.

Якщо інформація про приналежність завади $\xi$ деякому певному класу розподілів є відомою, то шляхом мінімізації оптимального критерію, що представляє собою узятий з оберненим знаком логарифм функції розподілу завади, може бути отримана оцінка максимальної правдоподібності ( $M$-оцінка). Якщо ж такої інформації немає, то для оцінювання шуканого вектору параметрів $\theta$ слід застосувати будь-який неквадратичний критерій, що забезпечує робастність одержуваної оцінки. Одним 3 таких критеріїв $є$ модульний критерій, що приводить до знакового алгоритму.

Аналіз останніх досліджень і публікацій. Застосування цього критерію в задачі ідентифікації об'єкта при наявності імпульсних завад розглядалося в [1-5]. Позитивні властивості модульного критерію використовуються в так званих комбінованих критеріях, найбільш поширеними серед яких є комбіновані функціонали, запропоновані Хьюбером [6] і Хемпелем [7]. Вони складаються 3 квадратичного функціоналу, що забезпечує оптимальність оцінок для гаусівського розподілу, і модульного, що дозволяє отримати більш робастну до розподілів з важкими “хвостами” (викидами) оцінку. Однак ефективність одержуваних робастних оцінок істотно залежить від численних параметрів, що використовуються в даних критеріях, і які обираються на основі досвіду дослідника [8-10]. 
Іншим підходом до отримання робастних оцінок, позбавлених зазначеного недоліку, є використання комбінованого критерію, заснованого на поєднанні квадратичного і модульного критеріїв більш простим способом, ніж запропонованих Хьюбером і Хемпелем.

Вперше такий критерій запропоновано в [11]. У роботах [12-15] даний критерій застосовувався для розв'язання задачі ідентифікації за наявністю імпульсних завад. В [16] був запропонований критерій найменшого четвертого ступеня, властивості якого вивчалися в роботах [17-21].

Комбінований критерій оцінювання для прискорення процесу ідентифікації, що використовує поєднання квадратичного критерію і критерію четвертого ступеня, запропонованого і дослідженого в [22], було розвинуто в роботі [23], метою якої було прискорення процесу ідентифікації. Властивості адаптивного алгоритму мінімізації такого комбінованого критерію вивчалися в роботі [24].

Як свідчить аналіз робіт, присвячених робастній ідентифікації об'єктів керування, застосування комбінованого критерію $є$ досить ефективним та значно простішим за використання традиційних критеріїв. У зв'язку з цим представляється вельми актуальним розробка підходу до робастного оцінювання параметрів з використанням деякого комбінованого функціоналу, що дозволяє поєднувати переваги МНК і МНМ.

\section{Виклад основного матеріалу}

\section{Дослідження збіжності робастної процедури ідентифрікації}

Для забезпечення робастних властивостей одержуваних оцінок досить ефективним є застосування комбінованого функціоналу навчання

$$
F[e(k)]=\frac{1}{4} \lambda e^{4}(k)+(1-\lambda)|e(k)|,
$$

де

$$
e(k)=y(k)-\hat{y}(k)+\xi(k)=y(k)-\hat{\theta}^{T}(k-1) x(k)+\xi(k),
$$

$\hat{y}(k) \quad$ - вихідний сигнал моделі;

$\hat{\theta}(k-1)=\left(\hat{\theta}_{1}(k-1), \hat{\theta}_{2}(k-1), . . \hat{\theta}_{N}(k-1)\right)^{T} \quad-\quad$ вектор оцінюваних параметрів $N \times 1 ; \lambda \in[0,1]$ - параметр змішування.

При використанні комбінованого критерію (2) градієнтна процедура мінімізації має вигляд

$$
\theta(k)=\theta(k-1)+\gamma(k)\left[\lambda e^{3}(k)+(1-\lambda) \operatorname{sign} e(k)\right] x(k),
$$

де $\gamma$ - деякий параметр, що впливає на швидкість збіжності алгоритму.

Дана процедура поєднує властивості МНК 3 властивостями МНМ, тому що при $\lambda=1$ з (3) маємо алгоритм МНК, а при $\lambda=0$ - алгоритм МНМ, i дозволяє боротися з негаусівськими завадами. Bapiюючи параметр $\lambda$, можна змінювати властивості алгоритму.

Введемо в розгляд помилку оцінювання

$$
\tilde{\theta}(k)=\theta(k)-\hat{\theta}(k),
$$

що дозволяє записати вираз для $e(k)$ в такий спосіб:

$$
e(k)=\tilde{\theta}^{T}(k-1) x(k)+\xi(k)=e_{a}(k)+\xi(k),
$$

де $e_{a}(k)=\tilde{\theta}^{T}(k-1) x(k)$ - апріорна помилка ідентифікації.

У зв'язку 3 тим, що передбачається $\xi(k) \sim N\left(0, \sigma_{\xi}^{2}\right)$, маємо

$$
\left.M\left\{e^{2}(k)\right)\right\}=\sigma_{\xi}^{2}+\sigma_{x}^{2} M\left\{\|\tilde{\theta}(k)\|^{2}\right\},
$$

де $M\{\bullet\}$ - символ математичного сподівання; $\|\bullet\|-$ евклідова норма.

Розглянемо питання збіжності процедури (3) за відсутності завад. При дослідженні питань збіжності скористаємося підходом, який застосовувався в [2526]. 3 цією метою введемо функцію Ляпунова $\|\tilde{\theta}(k)\|^{2}$.

Запишемо алгоритм (3) щодо помилок ідентифікації $\tilde{\theta}(i)$ :

$$
\tilde{\theta}(k)=\tilde{\theta}(k-1)-\gamma\left[\lambda e^{3}(k)+(1-\lambda) \operatorname{sign} e(k)\right] x(k) .(7)
$$

Помноживши обидві частини (7) зліва на $\tilde{\theta}^{T}(k), з$ урахуванням того, що $e(k)=\tilde{\theta}^{T}(k-1) x(k)$, маємо

$$
\begin{aligned}
& \|\tilde{\theta}(k)\|^{2}=\|\tilde{\theta}(k-1)\|^{2}-2 \gamma \lambda e^{4}(k)-2 \gamma(1-\lambda) e(k) \operatorname{sign} e(k)- \\
& -\gamma^{2} \lambda^{2} e^{6}(k)\|x(k)\|^{2}+2 \gamma^{2} \lambda(1-\lambda) e^{3}(k) \operatorname{sign} e(k)\|x(k)\|^{2}+(8) \\
& +\gamma^{2}(1-\lambda)^{2}\|x(k)\|^{2} .
\end{aligned}
$$

3 (8) видно, що при $\gamma>0$ приріст функції Ляпунова

$$
\Delta V(k)=\|\tilde{\theta}(k)\|^{2}-\|\tilde{\theta}(k-1)\|^{2}
$$

буде негативним, якщо

$$
\begin{gathered}
\left(\lambda e^{4}(k)+2(1-\lambda)|e(k)|\right)> \\
>\gamma\left(\lambda e^{3}(k)+(1-\lambda)|e(k)|\right)^{2}\|x(k)\|^{2} .
\end{gathered}
$$

Таким чином, умова збіжності процедури (3) буде виконуватися, якщо параметр $\gamma$ задовольняє нерівності

$$
0<\gamma<\frac{2 e(k)}{\left(\lambda e^{3}(k)+(1-\lambda) \operatorname{sign} e(k)\right)\|x(k)\|^{2}} .
$$

Оптимальне значення параметра $\gamma$ визначаємо 3 рівняння, одержуваного шляхом диференціювання (8) по $\gamma$ і прирівнювання похідної нулю. Таким чином, 


$$
\gamma^{\text {onm }}=\frac{e(k)}{\left(\lambda e^{3}(k)+(1-\lambda) \operatorname{sign} e(k)\right)\|x(k)\|^{2}} .
$$

Дослідимо статистичні властивості процедури навчання (3) при наявності перешкод виміру, тобто $y^{*}(k)=\theta^{*} x(k)+\xi(k), \xi(k) \sim N\left(0, \sigma^{2}\right)$. Припустимо, що завада не корельована 3 корисними сигналами. Записавши (3) щодо помилок навчання, маємо (7).

Розглянемо математичне сподівання $M\{\tilde{\theta}(k)\}$. Усереднюючи обидві частини (7), отримуємо

$$
\begin{aligned}
& M\{\tilde{\theta}(k)\}=M\left\{\tilde{\theta}(k-1)-2 \gamma \lambda\left(\tilde{\theta}^{T}(k-1) x(k)+\xi(k)\right) x(k)-\right. \\
& \left.-\gamma(1-\lambda) \operatorname{sign}\left(\tilde{\theta}^{T}(k-1) x(k)\right) x(k)\right\}
\end{aligned}
$$

Легко бачити, що

$$
\begin{aligned}
& M\left\{\tilde{\theta}^{T}(k-1) x(k) x(k)\right\}=M\left\{x(k) x^{T}(k) \tilde{\theta}(k-1)\right\}= \\
& =M\left\{x(k) x^{T}(k)\right\} M\{\tilde{\theta}(k-1)\}=R_{X X} M\{\tilde{\theta}(k-1)\},
\end{aligned}
$$

де $R_{X x}$ - кореляційна матриця вхідного сигналу.

Детально возглянемо вираз $M\left\{x(k) \operatorname{sign}\left(\tilde{\theta}^{T}(k-1) x(k)\right) x(k)\right\}$. У випадку, якщо сигнал $x(k) \sim N\left(0, \sigma_{x}^{2}\right)$, маємо

$$
\begin{aligned}
& M\left\{x(k) \operatorname{sign}\left(\tilde{\theta}^{T}(k-1) x(k)\right)\right\}= \\
& =M\left\{M\left\{x(k) \operatorname{sign}\left(\tilde{\theta}^{T}(k-1) x(k)\right)\right\}\right\}= \\
& =M\left\{\sqrt{\frac{2}{\pi}} \frac{1}{\sigma_{e}} M\left\{x(k)\left(\theta^{T}(k-1) x(k)\right)\right\}\right\}= \\
& =M\left\{\sqrt{\frac{2}{\pi \sigma_{e}^{2}}} M\left\{x(k) x^{T}(k) \theta(k-1)\right\}\right\}= \\
& =\sqrt{\frac{2}{\pi \sigma_{e}^{2}}} M\left\{x(k) x^{T}(k) \theta(k-1)\right\}= \\
& =\sqrt{\frac{2}{\pi \sigma_{e}^{2}}} R_{x x} M\{\theta(k-1)\},
\end{aligned}
$$

де $\sigma_{e}-$ середньоквадратичне значення помилки $e(k)$.

Вираз (9) отримано $з$ використанням теореми Прайса [27], згідно 3 якою для двох випадкових гаусівських величин $x$ та $y$ з нульовими математичними сподіваннями справедливо

$$
M\{x \operatorname{sign} y\}=\sqrt{\frac{2}{\pi}} \frac{1}{\sigma_{y}} M\{x y\},
$$

де $\sigma_{y}$ - середньоквадратичне значення $y$.
3 урахуванням властивостей завади $M\{\xi(k) x(k)\}=0$ і виразів (8-9) маємо

$$
\begin{gathered}
M\{\tilde{\theta}(k)\}= \\
=\left\{I-3 \gamma \lambda \sigma_{e}^{2} R_{x x}-\gamma(1-\lambda) \beta R_{x x}\right\} M\{\tilde{\theta}(k-1)\},
\end{gathered}
$$

звідки випливає, що процедура (3) буде збігатися в середньому, якщо параметр $\gamma$ задовольняє нерівності

$$
0<\gamma<\frac{2}{\left(3 \lambda \sigma_{e}^{2}+(1-\lambda) \beta\right) \operatorname{tr} R_{x x}} .
$$

Тут $\beta=\sqrt{\frac{2}{\pi \sigma_{e}^{2}}} ; \operatorname{tr} R_{x X}-$ слід матриці $R_{X X}$.

3 огляду на властивості вхідних сигналів, можемо записати умову (16) наступним чином:

$$
0<\gamma<\frac{2}{\left(3 \sigma_{e}^{2} \lambda+(1-\lambda) \beta\right) N \sigma_{x}^{2}} .
$$

Для дослідження збіжності алгоритму в середньоквадратичному розглянемо функцію Ляпунова $M\left\{\|\tilde{\theta}(k)\|^{2}\right\}$.

Помноживши обидві частини (7) зліва на $\tilde{\theta}^{T}(k)$, отримаємо

$$
\begin{gathered}
\|\tilde{\theta}(k)\|^{2}=\|\tilde{\theta}(k-1)\|^{2}-2 \gamma \lambda e^{3}(k)\left(\tilde{\theta}^{T}(k-1) x(k)\right)- \\
-2 \gamma(1-\lambda) \tilde{\theta}^{T}(k-1) x(k) \operatorname{sign} e(k)+\gamma^{2} \lambda^{2} e^{6}(k)\|x(k)\|^{2}+ \\
+2 \gamma^{2} \lambda(1-\lambda) e^{3}(k) \operatorname{sign} e(k)\|x(k)\|^{2}+\gamma^{2}(1-\lambda)^{2}\|x(k)\|^{2} . \\
\text { В даний вираз входять такі величини } e^{3}(k) \text { та } \\
e^{6}(k), \text { які відповідно дорівнюють } \\
e^{3}(k)=\left(e_{a}(k)+\xi(k)\right)\left(e_{a}^{2}(k)+2 e_{a}(k) \xi(k)+\xi^{2}(k)\right) ; \\
e^{6}(k)=\left(e_{a}(k)+\xi(k)\right)^{6}= \\
=e_{a}^{6}(k)+5 e_{a}^{5}(k) \xi(k)+6 e_{a}(k) \xi^{5}(k)+ \\
+e_{a}^{4}(k) \xi^{2}(k)+ \\
+20 e_{a}^{3}(k) \xi^{3}(k)+15 e_{a}^{2}(k) \xi^{4}(k)+\xi^{6}(k) .
\end{gathered}
$$

Взявши від обох частин (8) математичне сподівання, маємо

$$
\begin{aligned}
& M\left\{\|\tilde{\theta}(k)\|^{2}\right\}==M\left\{\|\tilde{\theta}(k-1)\|^{2}\right\}- \\
& -2 \gamma \lambda M\left\{e^{3}(k) e_{a}(k)\right\}- \\
& -2 \gamma(1-\lambda) M\left\{e_{a}(k) \operatorname{sign} e(k)\right\}+ \\
& +\gamma^{2} \lambda^{2} M\left\{e^{6}(k)\|x(k)\|^{2}\right\}+ \\
& +2 \gamma^{2} \lambda(1-\lambda) M\left\{e^{3}(k) \operatorname{sign} e(k)\|x(k)\|^{2}\right\}+ \\
& +\gamma^{2}(1-\lambda)^{2} M\left\{\|x(k)\|^{2}\right\} .
\end{aligned}
$$


Розглянемо кожний доданок в правій частині (19) 3 урахуванням (18) і статистичних властивостей сигналів і завад. У нашому випадку

$$
\begin{gathered}
M\left\{e^{3}(k) e_{a}(k)\right\}=M\left\{\left(\tilde{\theta}^{T}(k-1) x(k)\right)^{4}\right\} \approx \\
\approx 3 \sigma_{x}^{4}\left(M\left\{\|\tilde{\theta}(k-1)\|^{2}\right\}\right)^{2} ; \\
M\left\{\left(\tilde{\theta}^{T}(k-1) x(k)\right)^{2}\right\} M\left\{\xi^{4}(k)\right\} \approx \\
\approx \sigma_{x}^{2} M\left\{\xi^{4}(k)\right\} M\left\{\|\tilde{\theta}(k-1)\|^{2}\right\} ; \\
M\left\{\left(\tilde{\theta}^{T}(k-1) x(k)\right)^{6}\|x(k)\|^{2}\right\} \approx \\
\approx(15 N+90) \sigma_{x}^{8}\left(M\left\{\|\tilde{\theta}(k-1)\|^{2}\right\}\right)^{3} ; \\
M\left\{\left(\tilde{\theta}^{T}(k-1) x(k)\right)^{4}\|x(k)\|^{2}\right\}= \\
=(3 N+2) \sigma_{x}^{4} M\left\{\xi^{4}(k)\right\} M\left\{\|\tilde{\theta}(k-1)\|^{2}\right\} ; \\
M\left\{\left(\tilde{\theta}^{T}(k-1) x(k)\right)^{2}\|x(k)\|^{2}\right\}=
\end{gathered}
$$

$M\left\{\|x(k)\|^{2} \xi^{6}\right\}=M\left\{\|x(k)\|^{2}\right\} M\left\{\xi^{6}\right\}=N \sigma_{x}^{2} M\left\{\xi^{6}\right\} ;$

$$
M\left\{\|x(k)\|^{2}\right\}=N \sigma_{x}^{2} .
$$

Вираз для $M\left\{\tilde{\theta}^{T}(k-1) x(k) \operatorname{sign}\left(\theta^{T}(k-1) x(k)\right)\right\}$ проаналізуємо за аналогією з (9):

$$
\begin{aligned}
& M\left\{\tilde{\theta}^{T}(k-1) x(k) \operatorname{sign}\left(\tilde{\theta}^{T}(k-1) x(k)\right)\right\}= \\
= & M\left\{M\left\{\tilde{\theta}^{T}(k-1) x(k) \operatorname{sign}\left(\tilde{\theta}^{T}(k-1) x(k)\right) \mid \tilde{\theta}(k-1)\right\}\right\}= \\
= & M\left\{\tilde{\theta}^{T}(k-1) \sqrt{\frac{2}{\pi \sigma_{e}}} M\left\{x(k) x^{T}(k) \tilde{\theta}(k-1) \mid \tilde{\theta}(k-1)\right\}\right\} \approx \\
\approx & M\left\{\tilde{\theta}^{T}(k-1) \beta \tilde{\theta}(k-1)\right\} R_{\text {хх }}= \\
= & \beta \operatorname{tr} R_{\text {хх }} M\left\{\|\tilde{\theta}(k-1)\|^{2}\right\}=\beta \sigma_{x}^{2} M\left\{\|\tilde{\theta}(k-1)\|^{2}\right\} . \\
& \text { Аналогічно отримуємо } \\
& M\left\{\tilde{\theta}^{T}(k-1) x(k) \operatorname{sign}\left(\tilde{\theta}^{T}(k-1) x(k)\right)\|x(k)\|^{2}\right\}= \\
= & 3 \beta \sigma_{x}^{4} M\left\{\|\tilde{\theta}(k-1)\|^{2}\right\} .
\end{aligned}
$$

При обчисленні $M\left\{\|\tilde{\theta}(k)\|^{2}\right\}$ враховано статистичні властивості завади, тобто $M(\xi(k))=M\left(\xi^{3}(k)\right)=M\left(\xi^{5}(k)\right)=0$.

Підстановка отриманих виразів в (19) і нескладні перетворення дають

$$
\begin{aligned}
& M\left\{\|\tilde{\theta}(k)\|^{2}\right\}= \\
& \left(\begin{array}{l}
\left.1-6 \gamma \lambda \sigma_{x}^{2} \sigma_{\xi}^{2}+15(N+2) \gamma^{2} \lambda^{2} \sigma_{x}^{4} M\left\{\xi^{4}(k)\right\}-\right) \times \\
-2 \gamma(1-\lambda) \beta \sigma_{x}^{2}
\end{array}\right) \\
& \times M\left\{\|\tilde{\theta}(k)\|^{2}\right\}+ \\
& +\left(\begin{array}{l}
15(3 N+12) \gamma^{2} \lambda^{2} \sigma_{x}^{6} \sigma_{\xi}^{2}+ \\
+6 \gamma^{2} \lambda(1-\lambda) \sigma_{x}^{4} \beta-6 \gamma \lambda \sigma_{x}^{4}
\end{array}\right) \times \\
& \times\left(M\left\{\|\tilde{\theta}(k-1)\|^{2}\right\}\right)^{2}+ \\
& +(15 N+90) \gamma^{2} \lambda^{2} \sigma_{x}^{8}\left(M\left\{\|\tilde{\theta}(k-1)\|^{2}\right\}\right)^{3}+ \\
& +\gamma^{2} \sigma_{x}^{2} N\left(\lambda^{2} M\left\{\xi^{6}(k)\right\}+(1-\lambda)^{2}\right) .
\end{aligned}
$$

Якщо алгоритм збігається, величина $M\left\{\|\tilde{\theta}(k)\|^{2}\right\}$ буде малою. Тому для аналізу сталого стану вираз (22) можна спростити, нехтуючи величинами $\left(M\left\{\|\tilde{\theta}(k-1)\|^{2}\right\}\right)^{2}$ та $\left(M\left\{\|\tilde{\theta}(k-1)\|^{2}\right\}\right)^{3}$ і обмежуючись розглядом величини

$$
\begin{aligned}
& M\left\{\|\tilde{\theta}(k)\|^{2}\right\}=\left(1-6 \gamma \lambda \sigma_{x}^{2} \sigma_{\xi}^{2}+\right. \\
& 15(N+2) \gamma^{2} \lambda^{2} \sigma_{x}^{4} M\left\{\xi^{4}(k)\right\}- \\
& \left.-2 \gamma(1-\lambda) \beta \sigma_{x}^{2}\right) M\left\{\|\left.\tilde{\theta}(k)\right|^{2}\right\} .
\end{aligned}
$$

3 (23) випливає, що процедура (3) буде збігатися в середньоквадратичному (приріст функції Ляпунова буде негативним) за виконання умови

$$
M\left\{\|\tilde{\theta}(\infty)\|^{2}\right\}=\frac{\gamma N\left[\lambda^{2} M\left\{\xi^{6}\right\}+(1-\lambda)^{2}\right]}{15 \gamma(N+2) M\left\{\xi^{4}\right\}-6 \lambda \sigma_{\xi}^{2}-2(1-\lambda) \beta},
$$

тобто якщо параметр $\gamma$ задовольняє нерівності

$$
0<\gamma<\frac{2\left(3 \lambda \sigma_{\xi}^{2}+(1-\lambda) \beta\right)}{15(N+2) \lambda^{2} \sigma_{x}^{2} M\left\{\xi^{4}(k)\right\}} .
$$

Оптимальне значення цього параметра, що забезпечує максимальну швидкість збіжності алгоритму, яке отримується шляхом вирішення рівняння 


$$
\frac{\partial M\left\{\|\tilde{\theta}(k)\|^{2}\right\}}{\partial \gamma}=0
$$

матиме такий вигляд:

$$
\gamma^{\text {opt }}=\frac{3 \lambda \sigma_{\xi}^{2}+(1-\lambda) \beta}{15(N+2) \lambda^{2} M\left\{\xi^{4}(k)\right\}} .
$$

Зі співвідношення (23) можна отримати вираз для асимптотичної помилки оцінювання

$$
M\left\{\|\tilde{\theta}(\infty)\|^{2}\right\}=\frac{\gamma N\left[\lambda^{2} M\left\{\xi^{6}\right\}+(1-\lambda)^{2}\right]}{15 \gamma(N+2) M\left\{\xi^{4}\right\}-6 \lambda \sigma_{\xi}^{2}-2(1-\lambda) \beta},
$$

тобто для забезпечення $\lim _{k \rightarrow \infty} M\left\{\|\tilde{\theta}(\infty)\|^{2}\right\}=0$ параметр $\gamma$ повинен обиратися змінним і 3 ростом $k$ прагнути до нуля, тобто задовольняти умовам Дворецького [28].

Підстановка (24) в (6) дає вираз для асимптотичної помилки ідентифікації

$$
M\left\{e^{2}(\infty)\right\}=\sigma_{\xi}^{2}+\frac{\sigma_{x}^{2} \gamma N\left[\lambda^{2} M\left\{\xi^{6}\right\}+(1-\lambda)^{2}\right]}{15 \gamma(N+2) M\left\{\xi^{4}\right\}-6 \lambda \sigma_{\xi}^{2}-2(1-\lambda) \beta} .
$$

\section{Моделювання}

Розглядалася задача ідентифікації стаціонарного лінійного об'єкта, описуваного рівнянням (1) 3 наступними параметрами:

$$
\theta^{*}=(-5 ;-9 ;-40 ;-68 ; 16 ; 32 ; 64 ; 128 ; 256)^{T} .
$$

В якості вхідного сигналу $x(k)$ вибиралися послідовності нормально розподілених величин $x(k) \sim \mathrm{N}(0 ; 1)$. При тестуванні робастності алгоритмів в вихідний сигнал об'єкта додавався незалежний шум с розподілом Релея $(\gamma=1.0)$. Гістограма такої завади показана на рис. 1.

Результати моделювання при різних завданнях параметра змішування $\lambda$ представлені на рис. 2-3. На рис. 2, а, б показано графіки налаштування параметрів моделі при виборі $\lambda=0,5$ та $\lambda=0,7$ відповідно, а на рис. 3, а, б - відповідні помилки ідентифікації.

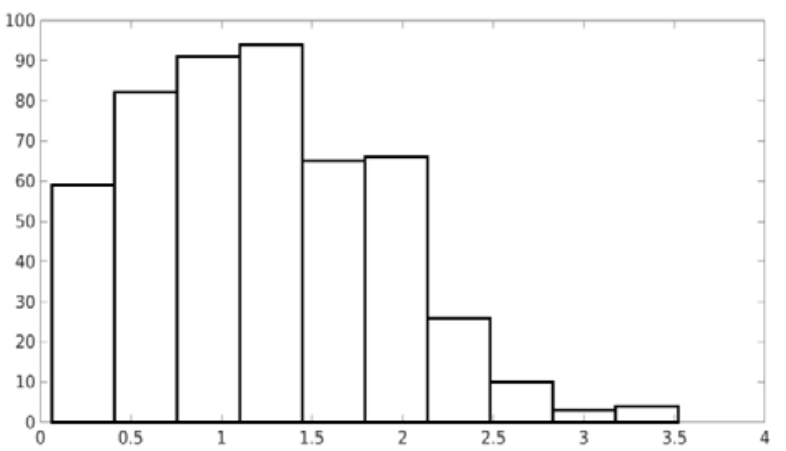

Рис. 1. Гістограма завади
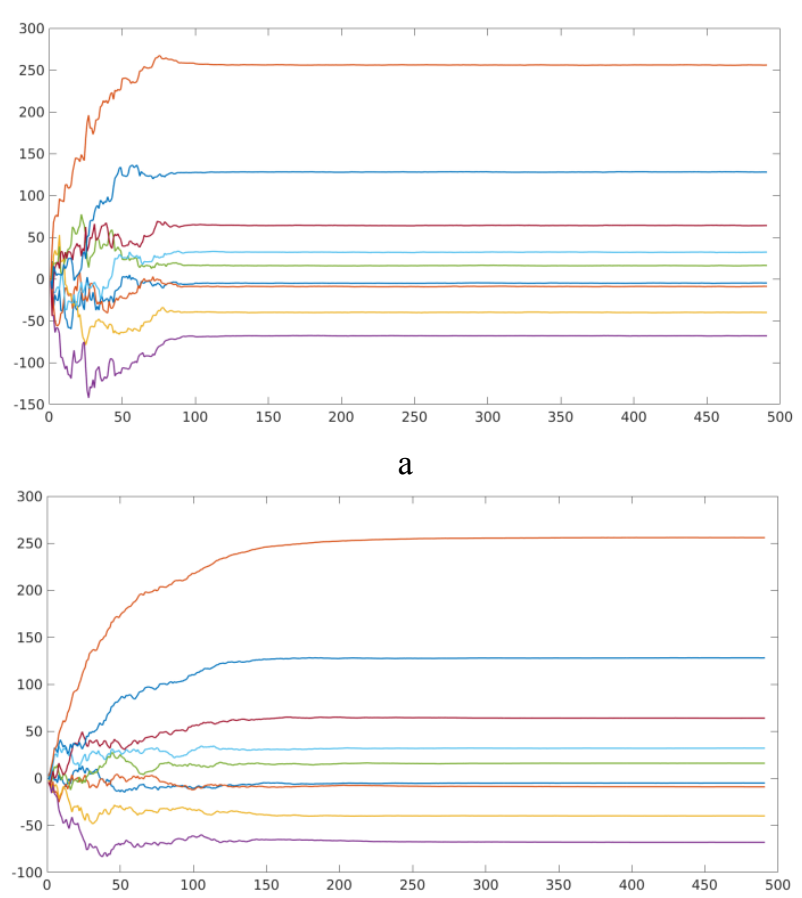

6

Рис. 2. Графік налаштування параметрів моделі
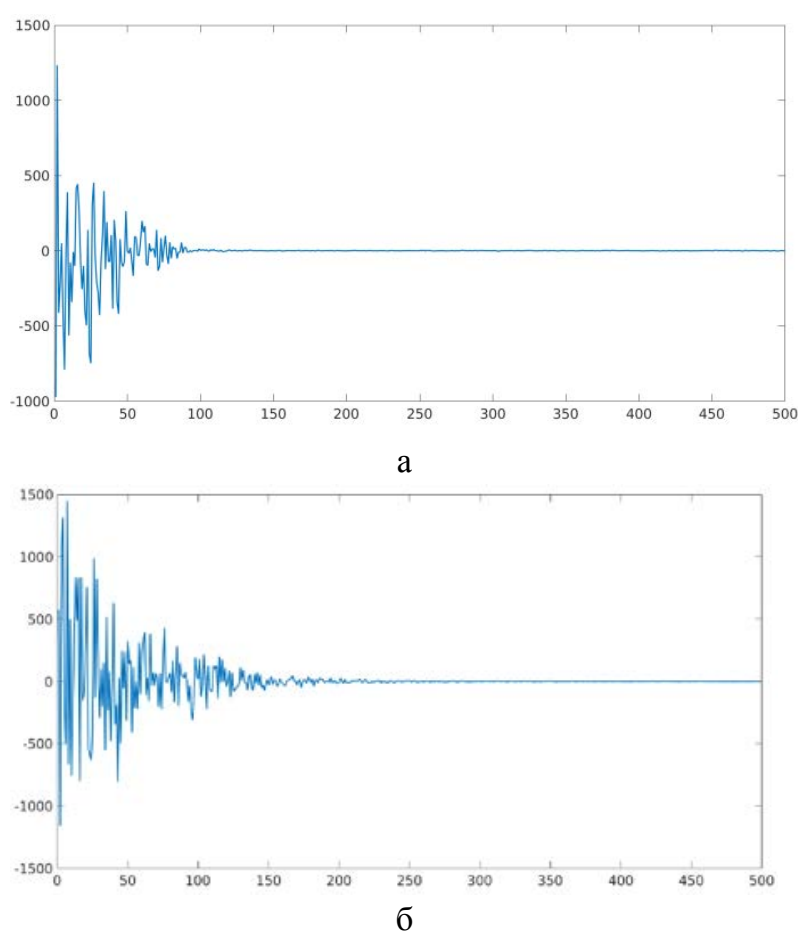

Рис. 3. Помилки ідентифікації

\section{Висновки}

В роботі був розглянутий новий метод ідентифікації лінійних об'єктів за умов негаусівських завад із застосуванням комбінованого функціоналу, який поєднує властивості критерію четвертого ступеня та методу найменших модулів. Визначено умови збіжності градієнтного алгоритму ідентифікації в середньому i середньоквадратичному. Отримано аналітичні оцінки неасимптотичних та асимптотич- 
них значень помилки оцінювання параметрів і точності ідентифікації. Показано, що ці значення помилки оцінювання і точності ідентифікації залежать від вибору параметра змішування. Отримані оцінки є досить загальними і залежать від статистичних характеристик корисних сигналів і завад. Тому для їх практичного застосування слід скористатися оцінками цих параметрів.

\section{Список літератури}

1. Shao T. An affine projection sign algorithm robust against impulsive interferences / T. Shao, Y.R. Zheng, J. Benesty // IEEE Signal Process. Lett. - 2010. - 17(4). - P. 327-330. https://doi.org/10.1109/LSP.2010.2040203.

2. Shin J. Variable step-size affine projection sign algorithm / J. Shin, J. Yoo, P. Park // Electronics Lett. - 2012. - 48(9). P. 483-485. https://doi.org/10.1049/el.2012.0751.

3. A novel normalized sign algorithm for system identification under impulsive noise interference / L. Lu, H. Zhao, K. Li, B. Chen // Int. J. of Electronics and Communications. - 2015. - 69(11). - P. 1590-1598. https://doi.org/10.1007/s00034-0150195-1.

4. Huang H. A new variable step-size NLMS algorithm and its performance analysis / H. Huang, J. Lee // IEEE Trans. Signal Process. - 2012. - 60(4). - P. 2055-2060. https://doi.org/10.1109/TSP.2011.2181505.

5. Casco-Sánchez F.M. A New Variable Step-Size NLMS Algorithm and its Performance Evaluation in Echo Cancelling Applications / F.M. Casco-Sánchez, R.C. Medina-Ramírez, M. López-Guerrero // J. of Applied Research and Technology. 2011. - 9. - 3. - P. 302-313. https://doi.org/10.22201/icat.16656423.2011.9.03.425.

6. Хьюбер П. Робастность в статистике / П. Хьюбер. - М.: Мир, 1984. - 304 с.

7. Hampel F.R. The influence curve and its role in robust estimation / F.R. Hampel // J. Amer. Statist. Assoc. - 1974. June. - 69. - P. 383-393. https://doi.org/10.2307/2285666.

8. Adamczyk T. Application of the Huber and Hampel M-estimation in Real Estate Value Modeling / T. Adamczyk // Geomatics and Environmental Engineering. - 2017. - 11. - pp. 15-23. https://doi.org/10.7494/geom.2017.11.1.15.

9. Zhao F. A Robust M-Shaped Error Weighted Algorithms for Censored Regression / F. Zhao, H. Zhao, W. Wang // Circuits, Systems, and Signal Processing. - 2020. - Vol. 39. - P. 324-343. https://doi.org/10.1007/s00034-019-01176.

10. A family of robust m-shaped error weighted least mean square algorithms: performance analysis and echo cancellation application / S. Zhang, W.X. Zheng, J. Zhang, H. Han / IEEE Access. - 2017. - P. 14716-14727. https://doi.org/10.1109/ACCESS.2017.2722464.

11. Chambers J. A Robust Mixed-Norm Adaptive Filter / J. Chambers, A. Avlonitis // IEEE Signal Processing Letters. 1997. - 4. - 2. - P. 46-48. https://doi.org/10.1109/97.554469.

12. Papoulis E.V. A Normalized Robust Mixed-Norm Adaptive Algorithm for System Identification / E.V. Papoulis, T. Stathaki // IEEE Signal Processing Letters. - 2004. - 11. - 1. - P. 56-59. https://doi.org/10.1109/LSP.2003.819353.

13. Arenas-Garcia J. Adaptive combination of normalised filters for robust system identification / J. Arenas-Garcia, A.R. Figueiras-Vidal // Electronics Lett. - 2005. - 41(15). - P. 874-875. https://doi.org/10.1049/el:20051936.

14. Robust identification of non-stationary objects with nongaussian interference / O. Rudenko, O. Bezsonov, V. Lebediev, N. Serdiuk // Eastern-European Journal of Enterprise Technologies. - 2019. - № 5/4(101). - P. 44-52. https://doi.org/10.15587/1729-4061.2019.181256.

15. Rakesh P. Modified least-mean mixed-norm algorithms for adaptive spars system identification under impulsive noise environment / P. Rakesh, T.K. Kumar, F. Albu // 42 Int. Conf. on Telecommunications and Signal Processing (TSP), Budapesht, July, 2019. - 1.- P. 557-561. https://doi.org/10.1109/TSP.2019.8768813.

16. Walach, E. The least mean fourth (LMF) adaptive algorithm and its family / E. Walach, D. Widrow // IEEE Trans. 1984. - IT-30. - P. 275-283. https://doi.org/10.1109/TIT.1984.1056886.

17. Bershad N. Mean-square stability of the normalized least mean fourth algorithm for white Gaussian Inputs / N. Bershad, J.C.M. Bermudez // Digit. Signal Process. - 2011. - 21(6). - P. 694-700. https://doi.org/10.1016/j.dsp.2011.06.002.

18. Eweda E. New insights into the normalization of the least mean fourth algorithm / E. Eweda, A. Zerguine // Signal Image Video Process. - 2013. - 7(2). - P. 255-262. https://doi.org/10.1007/s11760-011-0231-y.

19. Eweda E. Global stabilization of the least mean fourth algorithm / E. Eweda // IEEE Trans. Signal Process. - 2012. 60(3). - P. 1473-1477. https://doi.org/10.1109/TSP.2011.2177976.

20. Eweda E. Stochastic analysis of a stable normalized least mean fourth algorithm for adaptive noise canceling with a white Gaussian reference / E. Eweda, N. Bershad // IEEE Trans.Signal Process. - 2012. - 60(12). - P. 6235-6244. https://doi.org/10.1109/TSP.2012.2215607.

21. Hübscher P.I. A mean-square stability analysis of the least mean fourth adaptive algorithm / P.I. Hübscher, J.C.M. Bermudez, V.H. Nascimento // IEEE Trans. Signal Process. - 2007. - 55(8). - P. 4018-4028. https://doi.org/10.1109/TSP.2007.894423.

22. Chambers J. Least mean mixed-norm adaptive filtering / J. Chambers, O. Tanrikulu, A.G. Constantinides // Electronics letters. - 1994. - 30. - 19. - P. 1574-1575. https://doi.org/10.1049/el:19941060.

23. Zerguine A. A variable-parameter normalized mixed-norm (VPNMN) adaptive algorithm / A. Zerguine // EURASIP Journal on Advances in Signal Processing. - 2012. - 55. https://doi.org/10.1186/1687-6180-2012-55.

24. The Krylov-proportionate normalized least mean fourth approach: Formulationand performance analysis / M.O. Sayin, Y. Yilmaz, A. Demir, S.S. Kozat // Signal Process. - 2015. - Vol. 109. - P. 1-13. https://doi.org/10.1016/j.sigpro.2014.10.015. 
25. Analysis of convergence of adaptive single-step algorithms for the identification of non-stationary objects / O. Rudenko, O. Bezsonov, O. Romanyk, V. Lebediev // Eastern-European Journal of Enterprise Technologies. - 2019. - 1/4(97). - P. 6-14. https://doi.org/10.15587/1729-4061.2019.157288.

26. Руденко О.Г. Регуляризованный алгоритм обучения адалины в задаче оценивания нестационарных параметров / О.Г. Руденко, А.А. Бессонов // Управляющие системы и машины. - 2019. - № $1 . \quad$ - С. $22-30$. https://doi.org/10.15407/usim.2019.01.

27. Price R. A useful theorem for nonlinear devices having Gaussian inputs / R. Price // IREN Trans. Inform Theory. 1958. -4. - P. 69-72. https://doi.org/10.1109/TIT.1958.1057444.

28. Вазан М. Стохастическая аппроксимация / М. Вазан. - М.: Мир, 1972. - 295 с.

\section{References}

1. Shao, T., Zheng, Y.R. and Benesty, J. (2010), An affine projection sign algorithm robust against impulsive interferences, IEEE Signal Process. Lett., No. 17(4), pp. 327-330. https://doi.org/10.1109/LSP.2010.2040203.

2. Shin, J., Yoo, J. and Park, P. (2012), Variable step-size affine projection sign algorithm, Electronics Lett., No. 48(9), pp. 483-485. https://doi.org/10.1049/el.2012.0751.

3. Lu, L., Zhao, H., Li, K. and Chen, B. (2015), A novel normalized sign algorithm for system identification under impulsive noise interference, Int. J. of Electronics and Communications, No. 69(11), pp. 1590-1598. https://doi.org/10.1007/s00034015-0195-1.

4. Huang, H. and Lee, J. (2012), A new variable step-size NLMS algorithm and its performance analysis, IEEE Trans. Signal Process, No. 60(4), pp. 2055-2060. https://doi.org/10.1109/TSP.2011.2181505.

5. Casco-Sánchez, F.M., Medina-Ramírez, R.C. and López-Guerrero, M. (2011), A New Variable Step-Size NLMS Algorithm and its Performance Evaluation in Echo Cancelling, Applications of Applied Research and Technology, No. 9, Vol. 3, pp. 302-313. https://doi.org/10.22201/icat.16656423.2011.9.03.425.

6. Kh'yuber, P. (1984), “Robastnost' v statistike” [Robustness in statistics], Mir, Moscow, 304 p.

7. Hampel, F.R. (1974), The influence curve and its role in robust estimation, J. Amer. Statist. Assoc, June, No. 69, pp. 383-393. https://doi.org/10.2307/2285666.

8. Adamczyk, T. (2017), Application of the Huber and Hampel M-estimation in Real Estate Value Modeling, Geomatics and Environmental Engineering, No. 11, pp. 15-23. https://doi.org/10.7494/geom.2017.11.1.15.

9. Zhao, F., Zhao, H. and Wang, W. (2020), A Robust M-Shaped Error Weighted Algorithms for Censored Regression, Circuits, Systems, and Signal Processing, Vol. 39, pp. 324-343. https://doi.org/10.1007/s00034-019-01176.

10. Zhang, S., Zheng, W.X., Zhang, J. and Han, H. (2017), A family of robust m-shaped error weighted least mean square algorithms: performance analysis and echo cancellation application, IEEE Access, pp. 14716-14727. https://doi.org/10.1109/ACCESS.2017.2722464.

11. Chambers, J. and Avlonitis, A. (1997), A Robust Mixed-Norm Adaptive Filter, IEEE Signal Processing Letters, No. 4, Vol. 2, pp. 46-48. https://doi.org/10.1109/97.554469.

12. Papoulis, E.V. and Stathaki, T. (2004), A Normalized Robust Mixed-Norm Adaptive Algorithm for System Identification, IEEE Signal Processing Letters, No. 11, Vol. 1, pp. 56-59. https://doi.org/10.1109/LSP.2003.819353.

13. Arenas-Garcia, J. and Figueiras-Vidal, A.R. (2005), Adaptive combination of normalised filters for robust system identification, Electronics Lett., No. 41(15), pp. 874-875. https://doi.org/10.1049/el:20051936.

14. Rudenko, O., Bezsonov, O., Lebediev, V. and Serdiuk, N. (2019), Robust identification of non-stationary objects with nongaussian interference, Eastern-European Journal of Enterprise Technologies, No 5/4(101), pp. 44-52. https://doi.org/10.15587/1729-4061.2019.181256.

15. Rakesh, P., Kumar, T.K. and Albu, F. (2019), Modified least-mean mixed-norm algorithms for adaptive spars system identification under impulsive noise environment, 42 Int. Conf. on Telecommunications and Signal Processing (TSP), Vol. 1, Budapesht, pp. 557-561. https://doi.org/10.1109/TSP.2019.8768813.

16. Walach, E. and Widrow, D. (1984), The least mean fourth (LMF) adaptive algorithm and its family, IEEE Trans, IT 30, pp. 275-283. https://doi.org/10.1109/TIT.1984.1056886.

17. Bershad, N. and Bermudez, J.C.M. (2011), Mean-square stability of the normalized least mean fourth algorithm for white Gaussian Inputs, Digit. Signal Process, No. 21(6), pp. 694-700. https://doi.org/10.1016/j.dsp.2011.06.002.

18. Eweda, E. and Zerguine, A. (2013), New insights into the normalization of the least mean fourth algorithm, Signal Image Video Process, No. 7(2), pp. 255-262. https://doi.org/10.1007/s11760-011-0231-y.

19. Eweda, E. (2012), Global stabilization of the least mean fourth algorithm, IEEE Trans. Signal Process, No. 60(3), pp. 1473-1477. https://doi.org/10.1109/TSP.2011.2177976.

20. Eweda, E. and Bershad, N. (2012), Stochastic analysis of a stable normalized least mean fourth algorithm for adaptive noise canceling with a white Gaussian reference, IEEE Trans.Signal Process, No.60(12), pp. 6235-6244. https://doi.org/10.1109/TSP.2012.2215607.

21. Hübscher, P.I., Bermudez, J.C.M. and Nascimento, V.H. (2007), A mean-square stability analysis of the least mean fourth adaptive algorithm, IEEE Trans. Signal Process, No. 55(8), pp. 4018-4028. https://doi.org/10.1109/TSP.2007.894423.

22. Chambers, J., Tanrikulu, O. and Constantinides, A.G. (1984), Least mean mixed-norm adaptive filtering, Electronics letters, No. 30, Vol. 19, pp. 1574-1575. https://doi.org/10.1049/el:19941060.

23. Zerguine, A. (2012), A variable-parameter normalized mixed-norm (VPNMN) adaptive algorithm, EURASIP Journal on Advances in Signal Processing, No. 55. https://doi.org/10.1186/1687-6180-2012-55. 
24. Sayin, M.O., Yilmaz, Y., Demir, A. and Kozat, S.S. (2015), The Krylov-proportionate normalized least mean fourth approach: Formulationand performance analysis, Signal Process., $\quad$ Vol. 109, pp. 1-13. https://doi.org/10.1016/j.sigpro.2014.10.015.

25. Rudenko, O., Bezsonov, O., Romanyk, O. and Lebediev, V. (2019), Analysis of convergence of adaptive single-step algorithms for the identification of non-stationary objects, Eastern-European Journal of Enterprise Technologies, No. 1/4(97), pp. 6-14. https://doi.org/10.15587/1729-4061.2019.157288.

26. Rudenko, O.G. and Bezsonov, A.A. (2019), "Regulyarizovannyy algoritm obucheniya adaliny v zadache otsenivaniya nestatsionarnykh parametrov" [The regularized learning algorithm for adalins in the problem of estimating non-stationary parameters], Control systems and machines, No. 1, pp. 22-30. https://doi.org/10.15407/usim.2019.01.

27. Price, R. (1958), A useful theorem for nonlinear devices having Gaussian inputs, IREN Trans. Inform Theory. No. 4, pp. 69-72. https://doi.org/10.1109/TIT.1958.1057444.

28. Vazan, M. (1972), “Stokhasticheskaya approksimatsiya” [Stochastic approximation], Mir, Moscow, 295 p.

Надійшла до редколегії 30.01.2020 Схвалена до друку 10.03.2020

\section{Відомості про авторів:}

Руденко Олег Григорійович доктор технічних наук професор завідувач кафедри Харківського національного університету радіоелектроніки, Харків, Україна

https://orcid.org/0000-0003-0859-2015

Безсонов Олександр Олександрович доктор технічних наук доцент професор кафедри Харківського національного університету радіоелектроніки, Харків, Україна

https://orcid.org/0000-0001-6104-4275

\section{Сердюк Наталія Миколаївна} кандидат технічних наук доцент кафедри Харківського національного університету радіоелектроніки, Харків, Україна

https://orcid.org/0000-0002-0107-4365

\section{Олійник Кирило Олегович} аспірант Харківського національного університету радіоелектроніки, Харків, Україна https://orcid.org/0000-0001-8536-5217

\section{Романюк Олександр Сергійович} аспірант Харківського національного університету радіоелектроніки, Харків, Україна https://orcid.org/0000-0003-3278-1772

\section{Information about the authors:}

\section{Oleg Rudenko}

Doctor of Technical Sciences Professor

Head of Department of Kharkiv National

University of Radio Electronics,

Kharkiv, Ukraine

https://orcid.org/0000-0003-0859-2015

\section{Oleksandr Bezsonov}

Doctor of Technical Sciences Associate Professor

Professor of Kharkiv National

University of Radio Electronics,

Kharkiv, Ukraine

https://orcid.org/0000-0001-6104-4275

\section{Nataliia Serdiuk}

Candidate of Technical Sciences

Senior Lecturer of Kharkiv National

University of Radio Electronics,

Kharkiv, Ukraine

https://orcid.org/0000-0002-0107-4365

\section{Kirill Oliinyk}

Doctoral Student of Kharkiv National

University of Radio Electronics,

Kharkiv, Ukraine

https://orcid.org/0000-0001-8536-5217

\section{Oleksandr Romanyk}

Doctoral Student of Kharkiv National

University of Radio Electronics,

Kharkiv, Ukraine

https://orcid.org/0000-0003-3278-1772

\section{РОБАСТНАЯ ИДЕНТИФИКАЦИЯ ОБЪЕКТА НА ОСНОВЕ МИНИМИЗАЦИИ КОМБИНИРОВАННОГО ФУНКЦИОНАЛА}

О.Г. Руденко, А.А. Бессонов, Н.Н. Сердюк, К.О. Олейник, А.С. Романюк

Задача идентификации не только представляет интерес сама по себе, но и является составной частью общей задачи оптимизачии. Большинство существующих в настоящее время методов идентификации основано на использовании жестких и трудно проверяемых условий, связанных с гипотезой нормальности закона распределения помехи, $и$ таких, которые обосновываются ссылками на иентральную предельную теорему. Как известно, нормальным законом плотности распределения описываются помехи, присутствующие в измерениях, проводимых при абсолютной стабильности условий измерения, законом Лапласа, который имеет более длинные “хвосты”" - помехи, возникающие при максимальной нестабильности условий. Соответствующие алгоритмы идентификации в случае гауссовских помех основаны на методе наименьших квадратов (МНК), а в случае помех, распределенных по закону Лапласа, - на методе наименьших модулей (МНМ). Оба эти метода являются оптимальными в своих условиях и решения, получаемые с их помощью, могут сильно отличаться. Как показывает анализ работ, посвященных робастной идентификации объектов управления, применение комбинированного критерия, основанного на сочетании квадратичного и модульного критериев, обеспечивает робастность получаемых оченок, является весьма эффективным и значительно проще, чем использо- 
вание традиционных критериев. В связи с этим представляется весьма актуальным разработка подхода к робастному оиениванию параметров с использованием некоторого комбинированного функиионала, позволяет сочетать преимущества МНК и МНМ. В данной работе рассматривается задача идентификаиии параметров линейного объекта при наличии негаусовских помех на основе минимизации комбинированного функиионала, который сочетает свойства МНК и МНМ. Определены условия сходимости градиентного алгоритма идентификации в среднем и среднеквадратичном. Получены аналитические оценки неасимпотических и асимптотических значений ошибки оценивания параметров $и$ точности идентификации. Показано, что эти значения ошибки оченивания и точности идентификации зависят от выбора параметра смешивания. Для экспериментального исследования возможностей предлагаемого алгоритма производилась идентификация линейного объекта. При тестировании робастности алгоритмов в выходной сигнал объекта добавлялся независимый шум с распределением Рэлея. Результаты моделирования показали эффективность предложенного алгоритма при решении задачи идентификации линейного объекта при наличии негауссовских помех. В работе был рассмотрен новый метод идентификаиии линейных объектов в условиях негауссовских помех с применением комбинированного функиионала, который сочетает свойства МНК и МНМ. Определены условия сходимости градиентного алгоритма идентификачии в среднем и среднеквадратичном. Получены аналитические оценки неасимптотических и асимптотических значений ошибки оценивания параметров и точности идентификации. Показано, что эти значения ошибки оценивания и точности идентификации зависят от выбора параметра смешивания. Полученные оценки являются достаточно общими и зависят от статистических характеристик полезных сигналов и помех. Поэтому для их практического применения следует воспользоваться оценками этих параметров.

Ключевые слова: комбинированный критерий, градиентный алгоритм, весовой параметр, рекурсивная процедура, асимптотическая оценка, точность идентификации.

\section{WORKABLE IDENTIFICATION OF OBJECTS BASED ON MINIMIZATION OF COMBINED FUNCTIONAL}

O. Rudenko, O. Bezsonov, N. Serdiuk, K. Oliinyk, O. Romanyk

The identification task is not only of interest in itself, but also an integral part of the overall optimization task.

Most current methods of identification are based on the use of rigid and difficult to test conditions associated with the hypothesis of normality of the law of noise distribution and justified by references to the central limit theorem. As is known, the normal law of the distribution density describes the noise presence in the measurements carried out under absolute stability of the measurement conditions, the Laplace law, which has longer "tails" - interferences occurring under the maximum instability of the conditions. Accordingly, the identification algorithms in the case of Gaussian noise are based on the least mean squares method (LMS), and in the case of noise, distributed by Laplace's law, on the least mean modules method (LMM). Both of these methods are optimal in their own conditions and the solutions that come with them may vary greatly.According to the analysis of works on robust identification of control objects, the use of a combined criterion based on the combination of quadratic and modular criteria, which ensures the robustness of the obtained estimates, is quite effective and much easier than traditional criteria. In this regard, it seems very relevant to develop an approach to robust parameter estimation using some combined functionality, which allows combining the advantages of MNCs and MNMs. This paper deals with the problem of identifying the parameters of a linear object under the presence of non-Gaussian noise based on the minimization of a combined functional that combines the properties of MNCs and MNMs. The conditions of convergence of the gradient algorithm of identification in the mean and the standard are determined. Analytical estimations of non-asymptotic and asymptotic values of parameter estimation error and identification accuracy were obtained. It is shown that these values of estimation error and identification accuracy depend on the choice of mixing parameter. For the experimental investigation of the capabilities of the proposed algorithm, the identification of a linear object was performed. When testing the robustness of the algorithms, an independent noise with the Rayleigh distribution was added to the output of the object. The simulation results showed the effectiveness of the proposed algorithm in solving the problem of a linear object identification in the presence of non-Gaussian interference. A new method of linear objects identification in the conditions of non-Gaussian interference with the use of combined functional, which combines the properties of MNCs and MNMs, was considered in the paper. The conditions of convergence of the gradient identification algorithm in the mean and the standard are determined. Analytical estimations of non-asymptotic and asymptotic values of parameters estimation error and identification accuracy were obtained. It is shown that these values of estimation error and identification accuracy depend on the choice of mixing parameter. The obtained estimates are quite general and depend on the statistical characteristics of the useful signals and interference. Therefore, for their practical application, estimates of these parameters should be used.

Keywords: Combined criterion, gradient algorithm, weights parameter, recursive procedure, asymptotic estimation, accuracy of identification. 